\title{
Investigation of Blood Pressure during the First Trimester of Pregnancy in relation to the MTHFR C677T Polymorphism
}

\author{
E. Psara $^{1}$, E. O’Sullivan ${ }^{1}$, K. Pentieva ${ }^{1}$, M. Ward ${ }^{1}$, G. Horigan ${ }^{1}$, J.J. Strain ${ }^{1}$, C.F. Hughes ${ }^{1}$, \\ I. Bendik ${ }^{2}$, L. Doherty ${ }^{3}$ K. Glackin ${ }^{4}$ and H. McNulty ${ }^{1}$ \\ ${ }^{1}$ Nutrition Innovation Centre for Food and Health, Ulster University, Coleraine, United Kingdom, \\ ${ }^{2}$ Dept. Human Nutrition and Health, DSM Nutritional Products Ltd., Kaiseraugst, Switzerland, \\ ${ }^{3}$ Causeway Hospital, The Northern Health and Social Care Trust, Coleraine, United Kingdom and \\ ${ }^{4}$ Altnagelvin Hospital, The Western Health and Social Care Trust, Derry, United Kingdom
}

\begin{abstract}
The common C677T polymorphism in the MTHFR gene encoding the folate-metabolising enzyme methylenetetrahydrofolate reductase is implicated in hypertension and hypertension in pregnancy. Hypertension affects up to $15 \%$ of all pregnancies and has been identified as a leading cause of maternal and neonatal morbidity and mortality worldwide. We previously reported higher systolic and diastolic blood pressure (BP) in non-pregnant women with the variant MTHFR 677TT genotype compared to CT/CC genotypes. In addition, randomised controlled trials (RCTs) in non-pregnant hypertensive adults from our Centre demonstrated that supplemental riboflavin (co-factor for MTHFR) lowers BP specifically in those with the TT genotype. However, the role of this common folate polymorphism and its interaction with riboflavin during pregnancy remains unclear. The aim of this study was to investigate the impact of MTHFR genotype and riboflavin status on BP in pregnancy. Data were generated from the ongoing Optimal Nutrition for the Prevention of Hypertension (OptiPREG) project. Pregnant women were recruited at the end of the first trimester from antenatal clinics in Northern Ireland and in the Republic of Ireland. Participants were screened for MTHFR genotype and BP was measured according to current clinical guidelines. Biomarker status of riboflavin was determined using the erythrocyte glutathione reductase activation coefficient (EGRac), a functional assay with higher EGRac values representing a lower status. Overall, 117 $(11.6 \%)$ participants were identified with the variant MTHFR 677TT genotype. Both systolic and diastolic BP decreased from $8^{\text {th }}$ to $16^{\text {th }}$ gestational week $(\mathrm{GW})$, however, this typical BP pattern was not observed in the TT genotype group. After adjusting for maternal age, GW and body mass index, women with the TT genotype at $12^{\text {th }} \mathrm{GW}$ had higher mean systolic $(P 0.035)$ and diastolic $(P 0.034) \mathrm{BP}$. When the results at the $12^{\text {th }} \mathrm{GW}$ were stratified by riboflavin status, the BP phenotype owing to this polymorphism was evident only among women with lower status (i.e. EGRac >1.30), with mean (SEM) systolic BP of 120.4 (3.1) mmHg compared to $112.6(2.5) \mathrm{mmHg}$ in those with higher status (EGRac $\leq 1.30)$ within the TT genotype group; in contrast, low versus high riboflavin status had no impact on BP in CT/CC genotype groups. These results suggest that MTHFR genotype influences BP during pregnancy and that riboflavin can exert an important modulating effect on BP in women with TT genotype. An RCT is required to fully investigate the role of $M T H F R$ genotype and its interactive effect with riboflavin in BP during pregnancy.
\end{abstract}

\section{Conflict of Interest}

There is no conflict of interest. 